\title{
Downregulation of miR-146a inhibits osteoporosis in the jaws of ovariectomized rats by regulating the Wnt/ $\beta$-catenin signaling pathway
}

\author{
HUA LIU $^{1 *}$, XIANHU YUE ${ }^{1,2^{*}}$ and GANG ZHANG ${ }^{1}$ \\ ${ }^{1}$ Department of Orthopedics, The 960th Hospital of The PLA Joint Logistics Support Force, Jinan, Shandong 250031; \\ ${ }^{2}$ Department of Orthopedics, Jinan Central Hospital Affiliated to Shandong University, Jinan, Shandong 250013, P.R. China
}

Received July 28, 2020; Accepted November 5, 2020

DOI: $10.3892 /$ ijmm.2020.4839

\begin{abstract}
MicroRNAs (miRNAs or miRs) play important roles in osteoporosis and exhibit high potential in the therapeutic treatment of this condition. The present study aimed to explore the effects of miR-146a on bone loss noted in the jawbones of ovariectomized (OVX) rats and the interaction of miR-146a with the Wnt/ $\beta$-catenin signaling pathway. OVX Sprague-Dawley female rats were used to establish the animal model of osteoporosis (OP). Bone mineral density (BMD) was measured via dual-energy X-ray and the miR-146a levels were detected by reverse transcription-quantitative PCR. miR-146a antagonist (miR-146a-A) and negative control (miR-146a-NC) were used to examine the effects of miR-146a on OVX rats. The contents of osteocalcin and tartrate resistant phosphatase (TRAP) were detected via ELISA. Hematoxylin and eosin, and TRAP staining were used to observe the pathological changes and the number of osteoclasts in the jawbone, respectively. In addition, the expression levels of the nuclear factor of activated T cells c1 (NFATc1), c-Fos and cathepsin K (CTK) in the jawbone were detected by immunohistochemistry, whereas the expression levels of osteoprotegerin, TRAP, dickkopf 1 , Wnt 2 and $\beta$-catenin in the same tissues were assessed by western blot analysis. The Wnt 2 activator (DKK2-C2) and inhibitor (endostatin) were used to examine the effects of miR-146a on the Wnt/ $\beta$-catenin pathway. The results indicated that the BMD was increased, whereas the contents of osteocalcin and TRAP were decreased in the miR-146a-A group compared with those noted in the OP or negative control groups $(\mathrm{P}<0.05)$. Although the trabecular
\end{abstract}

Correspondence to: Dr Gang Zhang, Department of Orthopedics, The 960th Hospital of The PLA Joint Logistics Support Force, 25 Shifan Road, Tianqiao, Jinan, Shandong 250031, P.R. China

E-mail: zhanggang_edu@126.com

${ }^{*}$ Contributed equally

Key words: microRNA-146a, ovariectomized rats, Wnt//-catenin signaling pathway bone area of the OP group was decreased, the conditions were improved in the miR-146a-A group. The number of osteoclasts was decreased in the miR-146a-A group compared with that noted in the OP group $(\mathrm{P}<0.05)$. The expression levels of NFATc1, c-Fos and CTK in the miR-146a-A group were decreased compared with those noted in the OP or negative control groups $(\mathrm{P}<0.05)$. Similar results were found following the comparison of the miR-146a-A group with the DKK2-C2 group. Taken together, these data demonstrated that miR-146a downregulation inhibited OP of the jawbone in OVX rats by activating the $\mathrm{Wnt} / \beta$-catenin signaling pathway.

\section{Introduction}

Osteoporosis (OP) is a progressive bone disease featured by a decrease in bone mass and density, regression of bone condition contributing to increased incidence of bone fracture and a series of serious bone-associated diseases $(1,2)$. Currently, OP has become a main public health concern, notably due to the increased aging population worldwide (3). Menopausal OP is a bone metabolism disorder featured by the reduction of bone mass, high fracture rate and chronic alterations in the bone structure. This condition is caused by an imbalance in intraosseous homeostasis (4-6). Although complex factors induce postmenopausal OP, estrogen-dependent bone resorption has become the major etiological factor (7). The current therapeutic methods for postmenopausal OP are mainly concentrated on the inhibition of the absorption and identification of anabolic agents, such as estrogen replacements. However, these treatments also exhibit several side-effects, including high gynecological oncology risk and hypocalcemia $(8,9)$. Therefore, it is of utmost significance to identify novel targets and treatment methods for the clinical treatment of postmenopausal OP.

MicroRNAs (miRNAs or miRs) are a type of non-coding RNAs, with a nucleotide length ranging from 19-25. miRNAs regulate gene expression by controlling post-transcriptional processes $(10,11)$. Multiple miRNAs exert different biological functions in various organisms. More importantly, it has been shown that miRNAs participate in bone-associated diseases $(12,13)$. In addition, previous studies have concentrated on the function of miR-146a on rheumatoid arthritis (RA) and 
on its involvement in the progression of certain tumors $(14,15)$. However, the underlying mechanisms of miR-146a in the inhibition of osteoclast formation and the potential therapeutic effects caused on OP remain unclear.

It has been shown that the Wnt/ $\beta$-catenin signaling pathway exerts a significant effect on OP and that the inhibition of this pathway can improve bone density in an ovariectomized (OVX) rat model $(16,17)$. The induction of the receptor activator of the nuclear factor kappa $B$ ligand contributes to the $\mathrm{Wnt} / \beta$-catenin pathway-mediated regulation of osteoclastogenesis in bone tissues. In the jawbones of OVX rats, the Wnt/ $\beta$-catenin pathway was activated by miR-141 (18). However, the interaction of miR-146a with the Wnt/ $\beta$-catenin pathway in the jawbones of OVX rats remains unclear.

Therefore, the present study aimed to assess the effects of miR-146a on the regulation of the $\mathrm{Wnt} / \beta$-catenin signaling pathway in the jawbone of OVX rats. In addition, the results indicated that miR-146a was a negative factor of OP. Although the OVX model does not strictly imitate postmenopausal $\mathrm{OP}$, the data reported in the present study provide evidence that miR-146a may be a potential therapeutic target for postmenopausal OP.

\section{Materials and methods}

Animals. A total of 54 female Sprague-Dawley rats (12 week old, weighing $250 \pm 30 \mathrm{~g}$ ) were purchased from the Beijing Weitong Lihua Experimental Animal Technology Co., Ltd., [license no. SCXK (Jing) 2016-0006]. The feeding environment was maintained under standard conditions, including temperature range at $23 \pm 2^{\circ} \mathrm{C}$, average humidity of $55 \pm 5 \%$, light and dark cycle of $12 \mathrm{~h}$ and free access to food and water. The animal experiments followed the National Institute of Health guidelines (NIH Pub. no. 85-23, revised 1996) and were reviewed and approved by the Animal Protection and Use Committee of the 960th Hospital of the PLA Joint Logistics Support Force.

Construction of $O P$ model. The experimental rats were anesthetized by $3 \%$ sodium pentobarbital $(50 \mathrm{mg} / \mathrm{kg})$ by intraperitoneal injection. A bilateral ovariectomy method was used to prepare the OP model, as previously described (17). Basically, the rats had both ovaries removed. The bone mineral density (BMD) of the rat jaws was measured by dual-energy $\mathrm{X}$-ray absorptiometry at 12 weeks following modeling in order to confirm whether the OP model was successfully established.

The rats were randomly divided into 4 groups $(n=6)$ as follows: i) The sham-operated (sham) group, ii) OP group, iii) miR-146a antagonist group (miR-146a-A) and iv) miR-146a antagonist negative control group (miR-146-NC). In the sham group, part of the adipose tissue near the ovaries was removed in the rats and saline was injected into the tail vein once a week, whereas in the OP group, both ovaries were removed in the rats and saline was injected into the tail vein once a week. In the miR-146a-A group, part of the adipose tissue near the ovaries was removed and miR-146a antagonist (Guangzhou RiboBio Co., Ltd.) was injected at a dose of $10 \mathrm{mg} / \mathrm{kg}$ into the tail vein once a week (18), whereas in the miR-146a-NC group, part of the adipose tissue near the ovaries was removed and the negative control (Guangzhou RiboBio Co., Ltd.) was injected into the tail vein once a week $(10 \mathrm{mg} / \mathrm{kg})$. The treatment administration was carried out each week for 12 weeks. At the end of the treatment, the rats were sacrificed using $3 \%$ sodium pentobarbital $(120 \mathrm{mg} / \mathrm{kg})$ by intraperitoneal injection. Blood samples from the abdominal aorta and the bone of the jaws were collected. Certain jawbones were fixed in $4 \%$ paraformaldehyde and $1 \mathrm{~mm}$ below the center of the epiphyseal line was selected as the region of interest for the analysis by dual-energy X-ray absorptiometry. The analysis resulted in the determination of BMD. Certain jawbones were stored at $-80^{\circ} \mathrm{C}$.

To further examine the effects of miR-146a on the Wnt/ $\beta$-catenin signaling pathway, another 30 rats were divided into 5 groups $(n=6)$ as follows: i) The sham group, ii) OP group, iii) miR-146a-A group, iv) Wnt activator group (DKK2-C2) and v) miR-146a antagonist + Wnt inhibitor (endostatin) group $(\mathrm{miR}+\mathrm{E})$. The DKK2-C2 group was established by an injection of recombinant DKK 2 protein (20 $\mu \mathrm{g} / \mathrm{kg}$ DKK2-C2; Prospec-Tany TechnoGene, Ltd.) into the tail vein once a week (18). The miR $+\mathrm{E}$ group was treated with the miR-146-A, the Wnt inhibitor and recombinant human endostatin (Endostar, Shandong Xiansheng Mai Dejin Biopharmaceutical; $1.5 \mathrm{mg} / \mathrm{kg}$ ) that were injected into the tail vein once a week (18).

Reverse transcription-quantitative PCR (RT-qPCR). The mandibular molars of the rats $(100 \mathrm{mg})$ were homogenized using a homogenizer (KZ-II, Servicebio) until there was no visible tissue mass, then centrifuged at $4^{\circ} \mathrm{C}(800 \mathrm{x} \mathrm{g}, 15 \mathrm{~min})$. Total RNA was extracted using TRIzol reagent (Takara Bio, Inc.). cDNA was synthesized using the reverse transcription kit (Applied Biosystems; Thermo Fisher Scientific, Inc.). A Mastercycler ${ }^{\circledR}$ nexus X2 (Eppendorf) was used for qPCR. SYBR-Green PCR kit (Qiagen, Inc.) was used as the fluorophore. The following conditions were used: $95^{\circ} \mathrm{C}$ for $15 \mathrm{sec}$, $60^{\circ} \mathrm{C}$ for $60 \mathrm{sec}$ and $72^{\circ} \mathrm{C}$ for $40 \mathrm{sec}(35$ cycles). The data were processed using the $2^{-\Delta \Delta \mathrm{Cq}}$ method (19) and the relative expression levels were calculated using U6 mRNA as an internal reference. The sequences of the primers (Shanghai Biotech Engineering Services Co., Ltd.) were the following: miR-146a forward, 5'-CCTGAGAAGTGAATTCCATGGG-3' and reverse: 5'-TGGTGTCGTGGAGTCG-3'; and U6 forward, 5'-ATTGGA ACGATACAGAGAAGATT-3' and reverse, 5'-GGAACGCTTCACGAATTTG-3'.

ELISA. The serum samples were prepared by centrifugation $\left(800 \times \mathrm{g}, 10 \mathrm{~min}, 4^{\circ} \mathrm{C}\right)$ of the collected blood samples at $4^{\circ} \mathrm{C}$. The osteocalcin concentration was measured according to the instructions provided by the manufacturer (Rat ELISA kit; E4764-100; BioVision, Inc.). Tartrate-resistant acid phosphatase (TRAP) activity was determined using the TRAP ELISA kit (RA20761; Bio-Swamp Life Science Lab).

Hematoxylin and eosin $(H \& E)$ and TRAP staining. The molar area of the mandible of the rats was obtained and stored in a refrigerator at $-80^{\circ} \mathrm{C}$. The other part was fixed in a $4 \%$ paraformaldehyde solution for 2 days and was subsequently transferred to $10 \%$ EDTA for 2 months for decalcification. The mandible was dehydrated and paraffin-embedded and fixed with $4 \%$ paraformaldehyde at $37^{\circ} \mathrm{C}$ for $48 \mathrm{~h}$. The embedded tissue 
was sectioned to $5-\mu \mathrm{m}$-thick slices. The slices were routinely dewaxed and hydration, subsequently stained for $5 \mathrm{~min}$ in hematoxylin at room temperature (Beijing Solarbio Science $\&$ Technology Co., Ltd.). The sections were differentiated in hydrochloric acid and ethanol for $30 \mathrm{sec}$, immersed in PBS for $15 \mathrm{~min}$ and finally placed in eosin staining solution (Beijing Solarbio Science \& Technology Co., Ltd.) for $2 \mathrm{~min}$ at room temperature. They were routinely dehydrated, processed to form a transparent structure and mounted. The histopathological changes of the jawbone were photographed under a light microscope at x200 magnification (Olympus Corporation). The steps for the paraffin-embedded, dewaxed and hydrated sections were the same as those for H\&E staining. The sections were stained with the TARP staining kit (Whatman plc; GE Healthcare Life Sciences) for $1 \mathrm{~min}$ at $37^{\circ} \mathrm{C}$. The sections were dehydrated with gradient ethanol, cleared with dimethylbenzene and sealed with neutral balsam.

Immunohistochemistry. The slices were baked at $60^{\circ} \mathrm{C}$ in a dryer (101-1A, Nanjing Wohuan Science \& Technology Industrial Co. Ltd.) for $120 \mathrm{~min}$ and dewaxed with xylene, and sequentially hydrated with a gradient ethanol solution. A $3 \% \mathrm{H}_{2} \mathrm{O}_{2}$ methanol solution was used to inactivate processing for $20 \mathrm{~min}$. The citrate buffer ( $\mathrm{pH} \mathrm{6.0)}$ was heated for $10 \mathrm{~min}$ and sealed with 5\% BSA for $20 \mathrm{~min}$. Rabbit anti-rat nuclear factor of activated T cells c1 (NFATc1); 1:100, sc-17834, Santa Cruz Biotechnology, Inc.), c-Fos antibody (1:100, ab209794; Abcam) and cathepsin K (CTK) antibody (1:100, ab19027; Abcam) were incubated with the samples at $4^{\circ} \mathrm{C}$ overnight. Goat anti-rabbit IgG (1:1,000, ABIN101988; antibodies-online Aachen) labeled with horseradish peroxidase was used for secondary antibody incubation at room temperature for $60 \mathrm{~min}$. The chromogenic reaction was detected using DAB. Following DAB staining, the sections were re-dyed and dehydrated with gradient ethanol solution. Subsequently, the sections were transparently treated with xylene and sealed with neutral gum. The visual inspection was made under an optical microscope at x200 magnification (Olympus, Corporation). A total of 3 fields were randomly selected from each section and image analysis was performed using ImageJ 6.0 software, which was used to measure the integral optical density.

Western blot analysis. The tissues were homogenized and the supernatant was obtained following centrifugation $(800 \mathrm{x} \mathrm{g}$, $10 \mathrm{~min})$ at room temperature. The protein concentration was measured using the BCA kit (Beijing Solarbio Science $\&$ Technology Co., Ltd.). A total of $40 \mu \mathrm{g}$ protein sample was mixed with $10 \%$ SDS gel buffer and the protein was denatured by heating at $95^{\circ} \mathrm{C}$ for $5 \mathrm{~min}$. Subsequently, the proteins were subjected to $12 \%$ SDS-PAGE and transferred to PVDF membranes, which were blocked with TBST solution containing $5 \%$ skimmed milk powder for $1 \mathrm{~h}$ at $4^{\circ} \mathrm{C}$. The following antibodies were used: Rabbit anti-rat osteoprotegerin (OPG; 1:300, ab203061; Abcam), TRAP (1:10,000, ab133288; Abcam), dickkopf1 (DKK1; 1:2,000, ab61275; Abcam), Wnt2 (1:1,000, ab27794; Abcam), $\beta$-catenin (1:8,000, ab32572, Abcam) and $\beta$-actin $(1: 2,000$, ab61275; Abcam). The polyclonal antibodies were diluted with TBST solution containing $3 \%$ bovine serum protein and incubated overnight at $4^{\circ} \mathrm{C}$. Goat anti-rabbit IgG (1:1,000, ABIN 101988; antibodies-online,
Aachen) labeled with horseradish peroxidase was incubated at room temperature for $1 \mathrm{~h}$ following pre-incubation. The PVDF membrane was incubated with the ECL substrate for 3-5 min following washing. The protein expression levels were normalized with $\beta$-actin and the analysis was performed using ImageJ $6.0(\mathrm{NIH})$ software.

Statistical analysis. SPSS19.0 statistical software was used to analyze the data. The results are expressed as the means \pm SD. The analysis between multiple groups was performed by the single factor analysis of variance and by the Tukey's test. A $\mathrm{P}<0.05$ was considered to indicate a statistically significant difference.

\section{Results}

Effects of miR-146a on the expression of osteocalcin and TRAP in the serum of $O V X$ rats. The BMD in the jawbones of the rats in the OP group was significantly decreased compared with that of the rats in the sham group (Fig. $1 \mathrm{~A}, \mathrm{P}<0.05$ ). However, the BMD was increased in the miR-146a-A group compared with that noted in the OP group $(\mathrm{P}<0.05)$. The expression levels of miR-146a were measured by RT-qPCR (Fig. 1B). The data indicated that the expression levels of miR-146a were significantly increased in the OP group compared to those of the sham group $(\mathrm{P}<0.05)$. The expression levels of miR-146a in the miR-146a-A group were notably decreased compared to those of the OP group $(\mathrm{P}<0.05)$. The contents of osteocalcin (Fig. 1C) and TRAP (Fig. 1D) were also analyzed in the serum samples derived from each group. The data demonstrated that the levels of osteocalcin and TRAP were elevated in the OP group compared to those of the sham group $(\mathrm{P}<0.05)$. However, the contents of osteocalcin and TRAP in the serum of the miR-146a-A group were significantly decreased compared to those of the OP or the miR-146a-NC groups $(\mathrm{P}<0.05)$. Taken together, these data indicated that inhibition of miR-146a attenuated OP.

Effects of miR-146a on the pathological changes of the jawbone. The results of $\mathrm{H} \& \mathrm{E}$ staining indicated that the morphology and structure of the bone trabeculae in the sham group were arranged regularly and the bone marrow cavity was relatively small (Fig. 2A). However, the structure of the bone trabeculae was sparsely arranged with poor connectivity and a large number of blind ends in the trabecular bone. The thickness of the bone trabecular wall was inconsistent and the bone marrow cavity was increased in the OP and miR-146a-NC groups. However, in the bone trabeculae were thick and the bone marrow cavity was reduced in the miR-146a-A group compared with that of the OP group. The number of osteoblasts in the OP and miR-146a-NC groups was significantly lower than that noted in the sham group $(\mathrm{P}<0.05)$. The inhibition of miR-146a expression significantly increased the number of osteoblasts compared with that noted in the OP group $(\mathrm{P}<0.05)$. TRAP staining (Fig. $2 \mathrm{~B})$ indicated that the number of osteoclasts in the OP group was higher than that in the sham group $(\mathrm{P}<0.05)$. The expression levels of TRAP in the OP and miR-146a-NC groups were significantly higher than those noted in the miR-146a antagonist group $(\mathrm{P}<0.05)$. Therefore, the results indicated that the downregulation of 
A

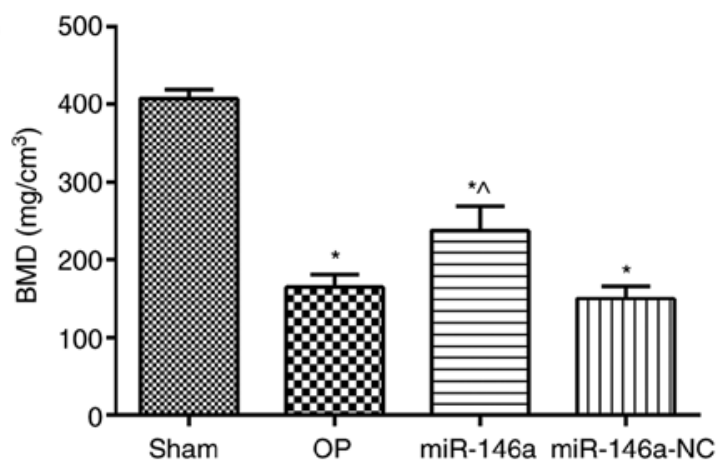

C

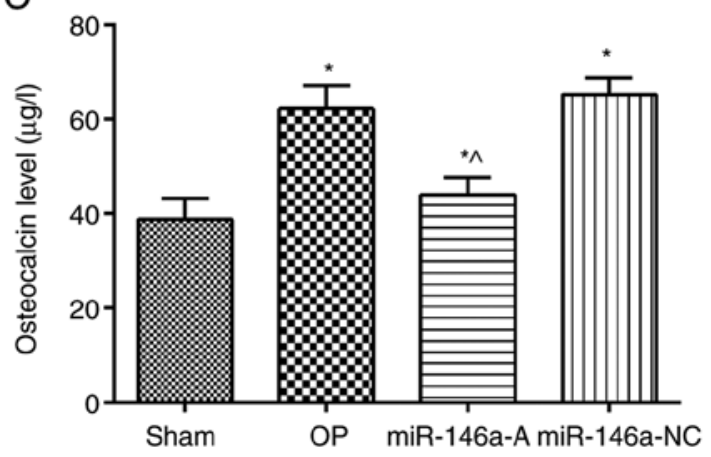

B

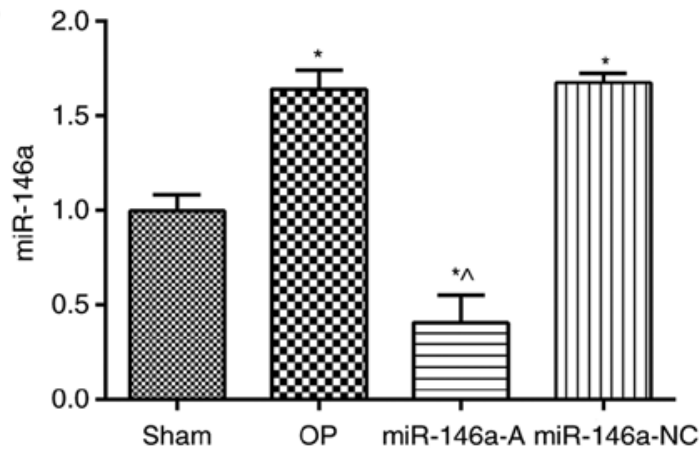

D

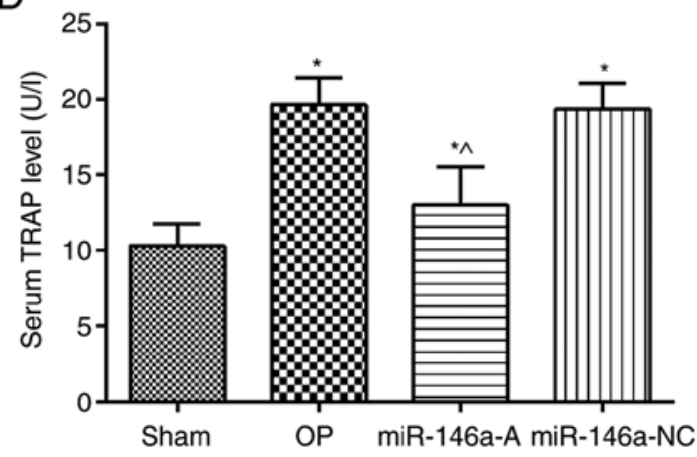

Figure 1. Evaluation of BMD, miR-146a, osteocalcin and TRAP expression levels. BMD and miR-146a levels were assessed in the jawbones of rats, whereas the expression levels of osteocalcin and TRAP were investigated in serum. (A) BMD. (B) miR-146a expression levels. (C) Osteocalcin levels. (D) Serum TRAP levels. ${ }^{*} \mathrm{P}<0.05$, compared with the sham group; ${ }^{\wedge} \mathrm{P}<0.05$, compared with the OP group. BMD, bone mineral density; miR-146a, microRNA-146a; TRAP, tartrate-resistant acid phosphatase; sham, sham group; OP, osteoporosis group; miR-146-A, miR-146a antagonist group; miR-146a-NC, miR-146a antagonist negative control group.

miR-146a attenuated the OP-associated pathological changes of the jawbone.

Effects of miR-146a on the expression of NFATc1, c-Fos and CTK in the jawbone. The expression levels of NFATc1, c-Fos and CTK in the jawbone were analyzed by immunohistochemistry (Fig. 3). The expression levels of NFATc1 (Fig. 3A), c-Fos (Fig. 3B) and CTK (Fig. 3C) in the jawbone tissues were significantly increased in the OP group compared to those of the sham group $(\mathrm{P}<0.05)$. However, the expression levels of NFATc1, c-Fos and CTK were significantly decreased in the miR-146a-A group $(\mathrm{P}<0.05)$. In addition, the levels of NFATc1, c-Fos and CTK were significantly increased in the miR-146a-NC group compared to those of the miR-146a-A group $(\mathrm{P}<0.05)$. Thus, the downregulation of miR-146a decreased the expression levels of NFATc1, c-Fos and CTK in the jawbone and relieved the effects of OP.

Effects of miR-146a on the protein expression of OPG, TRAP, $D K K 1$, Wnt 2 and $\beta$-catenin in the jawbone. The expression levels of OPG, Wnt 2 and $\beta$-catenin in the jawbone were examined by western blot analysis (Fig. 4). The data indicated that the expression levels of OPG, Wnt 2 and $\beta$-catenin were significantly decreased, while the expression levels of TRAP and DKK1 proteins were significantly increased in the OP group compared to those noted in the sham group $(\mathrm{P}<0.05)$. However, the expression levels of OPG, Wnt 2 and $\beta$-catenin proteins were significantly increased, while the expression levels of
TRAP and DKK1 proteins were significantly decreased in the miR-146a-A group compared to those noted in the OP group $(\mathrm{P}<0.05)$. The expression levels of OPG, Wnt 2 and $\beta$-catenin proteins were significantly decreased, while the expression levels of TRAP and DKK1 proteins were significantly increased in the miR-146a-NC group than those noted in the miR-146a-A group $(\mathrm{P}<0.05)$. These results indicated that the downregulation of miR-146a regulated the expression of $\mathrm{OP}$-associated proteins in the jawbone, which resulted in the reduction of the progression of OP.

miR-146a affects OP by regulating the Wnt/ $\beta$-catenin signaling pathway. To further assess the effects of miR-146a on the Wnt/ $\beta$-catenin signaling pathway, the Wnt 2 activator and inhibitor were used. The BMD of each group was analyzed (Fig. 5A). The BMD of the latter groups was significantly decreased compared with that of the sham group $(\mathrm{P}<0.05)$, whereas the BMD in the miR-146a-A and DKK2-C2 groups was significantly increased compared with that of the OP group $(\mathrm{P}<0.05)$. In addition, the BMD was significantly decreased following treatment of the cells with miR-146a-A and endostatin $(\mathrm{miR}+\mathrm{E})$ compared with that of the miR-146a-A group $(\mathrm{P}<0.05)$.

The contents of osteocalcin and TRAP in the serum of the rats in the other groups were significantly increased compared to those of the sham group ( $\mathrm{P}<0.05$; Fig. $5 \mathrm{~B}$ and $\mathrm{C})$. The levels of osteocalcin and TRAP in the serum of the miR-146a-A and DKK2-C2 group rats were significantly decreased 
A

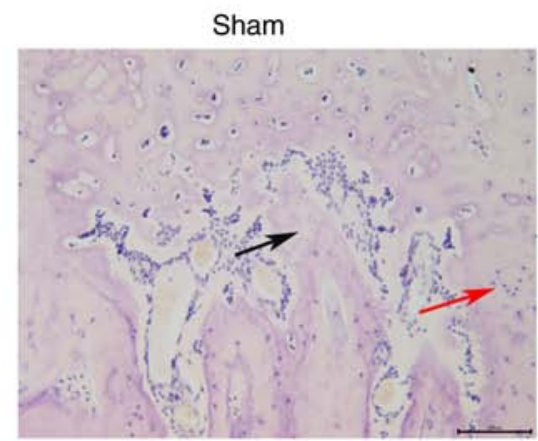

miR-146a-NC

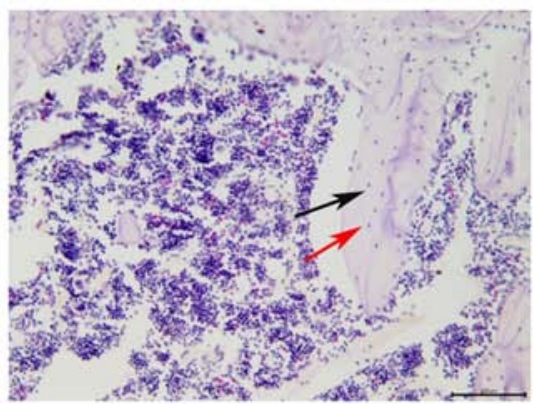

OP

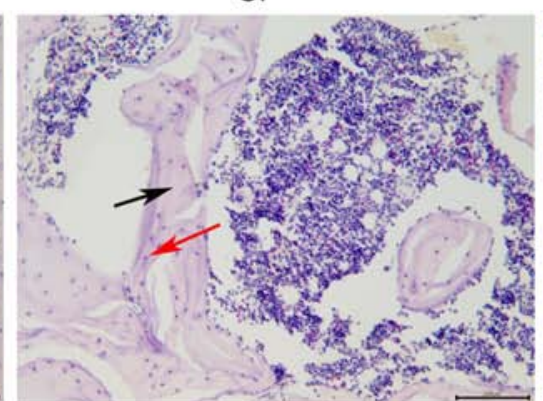

miR-146a-A

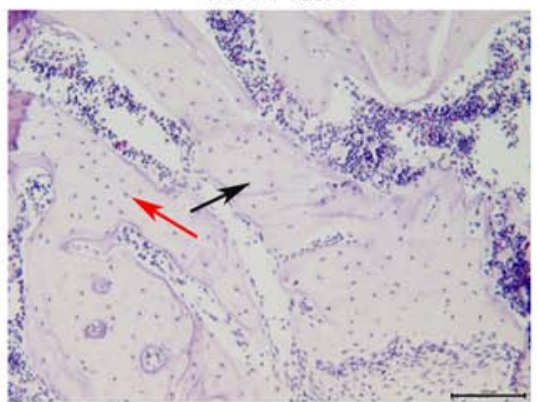

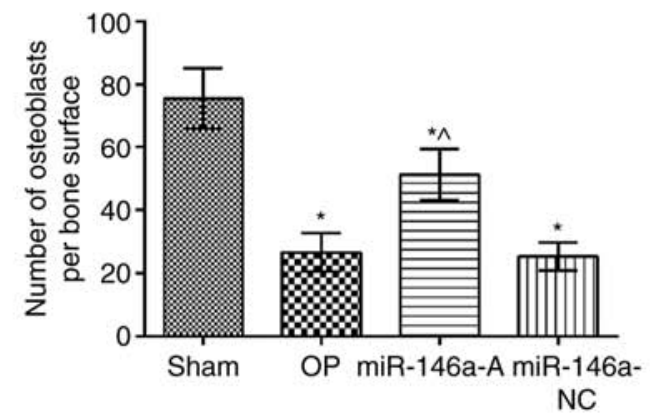

B

Sham

OP

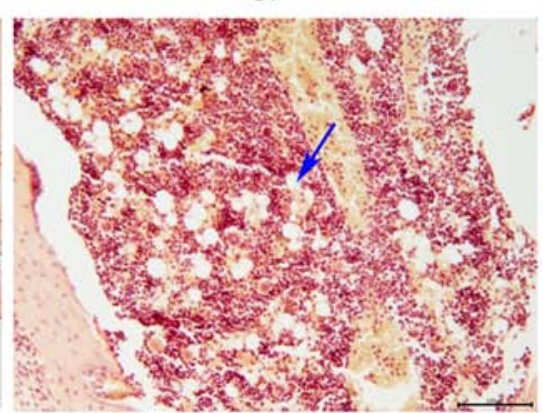

miR-146a-A

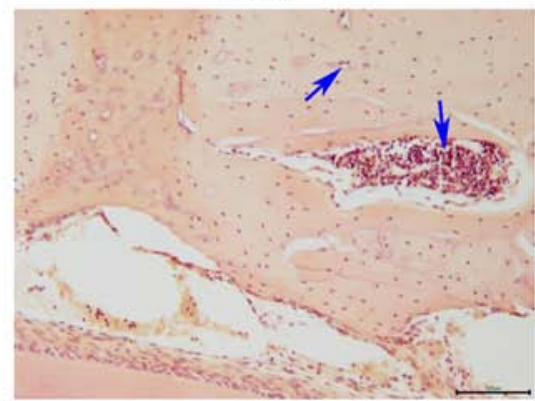

miR-146a-NC

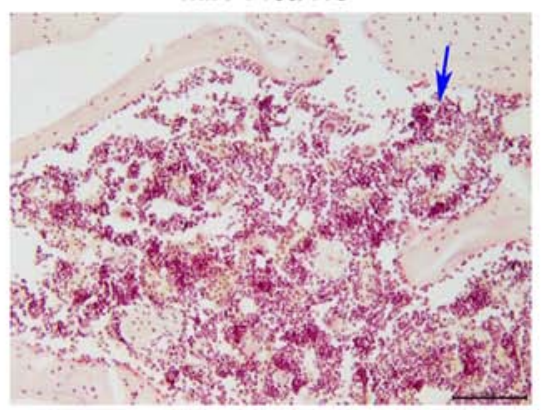

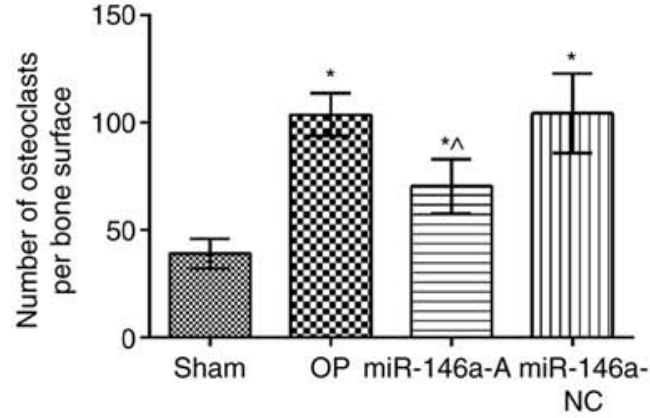

NC

Figure 2. Effects of miR-146a on the pathological alterations of the jaw and on TRAP expression. (A) Pathological alterations (x200 magnification). (B) Expression levels of TRAP x 200 magnification). Black arrows represent bone trabeculae. Red arrow represent osteoblasts. Blue arrows represent osteoclasts. "P $<0.05$, compared with the sham group; ${ }^{\wedge} \mathrm{P}<0.05$, compared with the OP group. miR-146a, microRNA-146a; TRAP, tartrate-resistant acid phosphatase; sham, sham group; OP, osteoporosis group; miR-146a-A, miR-146a antagonist group; miR-146a-NC, miR-146a antagonist negative control group.

compared to those of the OP group $(\mathrm{P}<0.05)$. However, the serum levels of osteocalcin and TRAP in the miR + E group were significantly increased compared to those of the miR-146a-A group $(\mathrm{P}<0.05)$.

The trabecular structure was complete and arranged tightly and regularly in the sham group, as determined by light microscopy (Fig. 5D). Moreover, the trabecular connections were meshed and the bone marrow cavity was relatively small, while the trabecular structure of the OP and $\mathrm{miR}+\mathrm{E}$ groups was sparse and its total content was significantly reduced. However, the trabeculae were thicker and the bone marrow cavity was decreased in the miR-146a antagonist and DKK2-C2 groups compared to those of the OP group. 
A
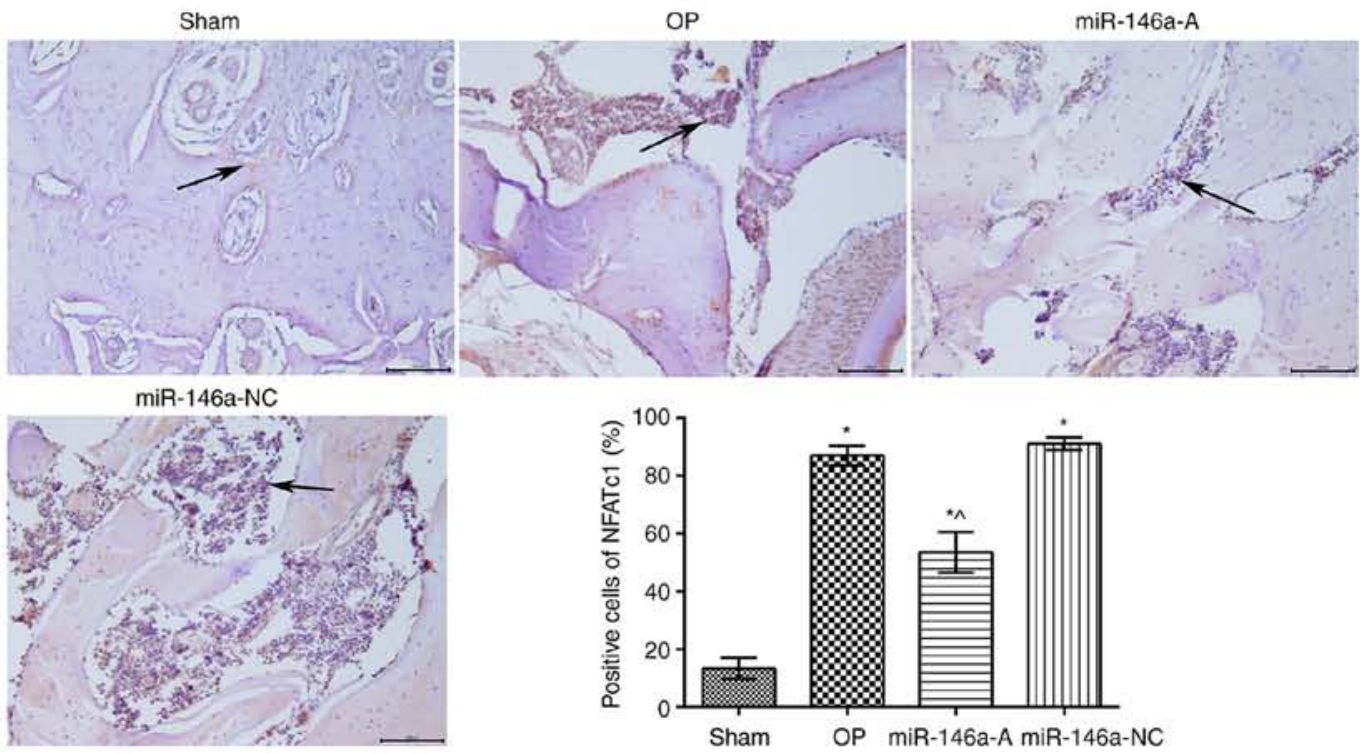

B

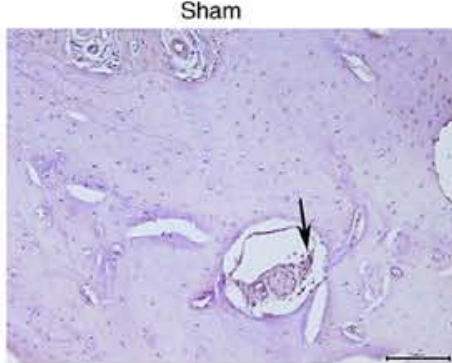

OP miR-146a-A
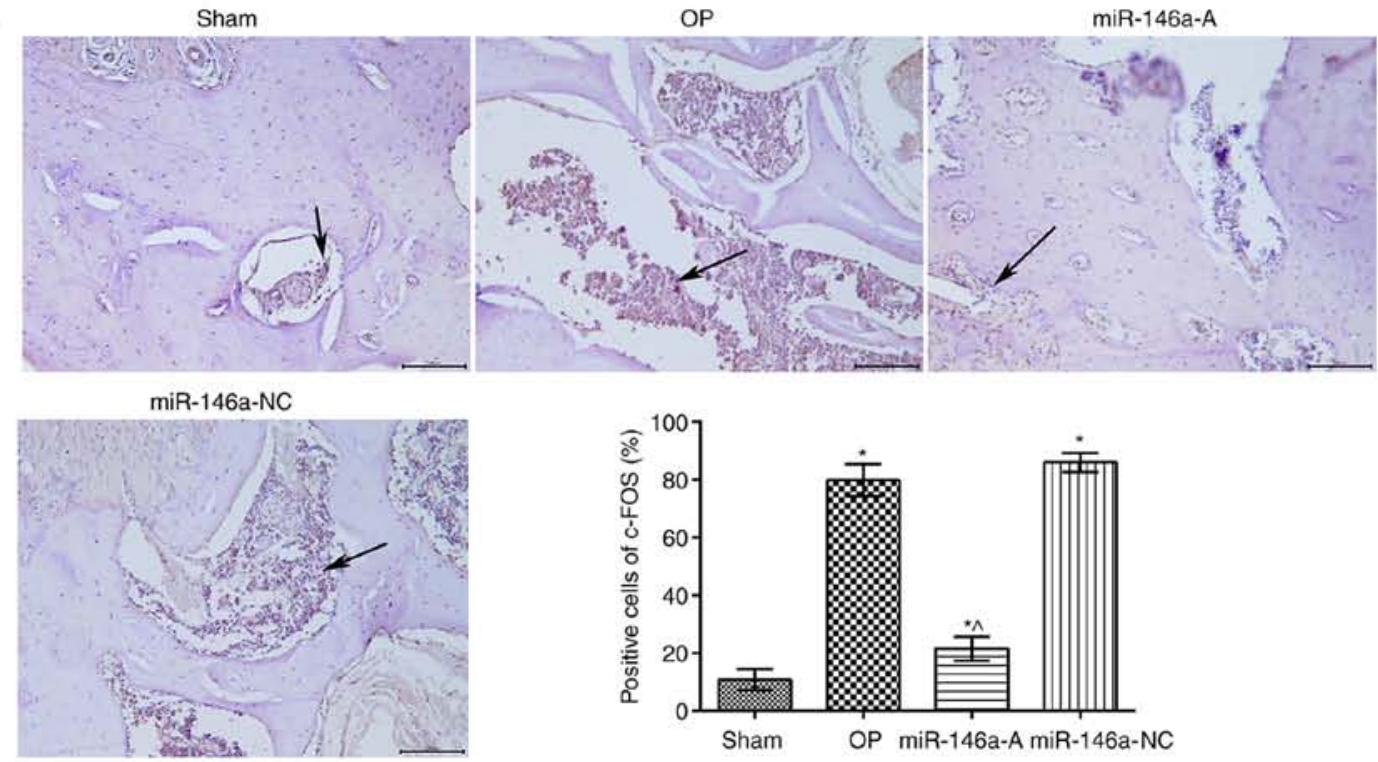

C

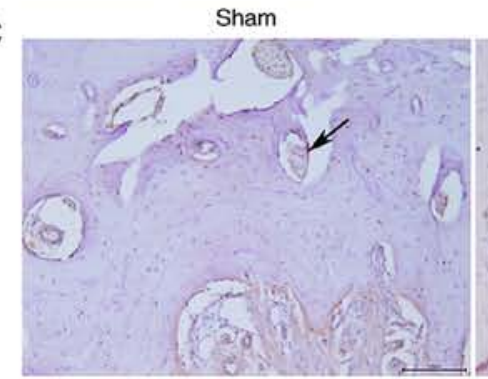

$\operatorname{miR}-146 a-N C$

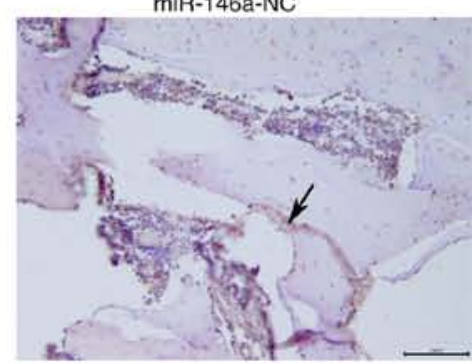

OP
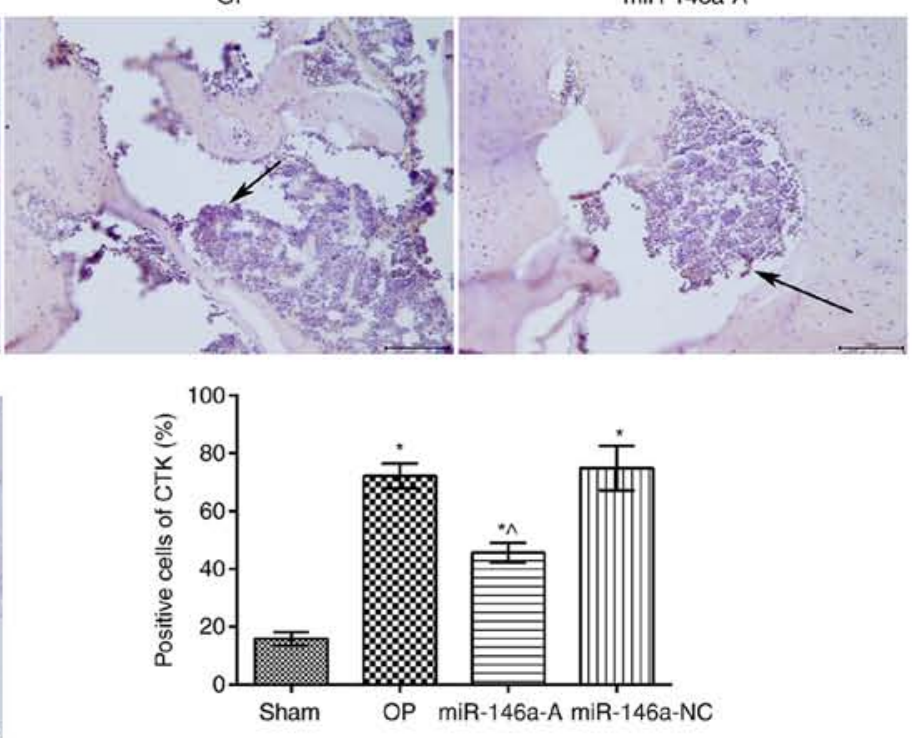

Figure 3. Effects of miR-146a were assessed on the expression of (A) NFATc1, (B) c-Fos and (C) CTK in rat jawbone tissues by immunohistochemical analysis (x200 magnification). Black arrows represent the expression levels determined by immunohistochemical analysis. * $\mathrm{P}<0.05$, compared with the sham group; ${ }^{\wedge} \mathrm{P}<0.05$, compared with the OP group. miR-146a, microRNA-146a; NFATc1, nuclear factor of activated T cells c1; CTK, cathepsin K; sham, sham group; OP, osteoporosis group; miR-146a-A, miR-146a antagonist group; miR-146a-NC, miR-146a antagonist negative control group. 

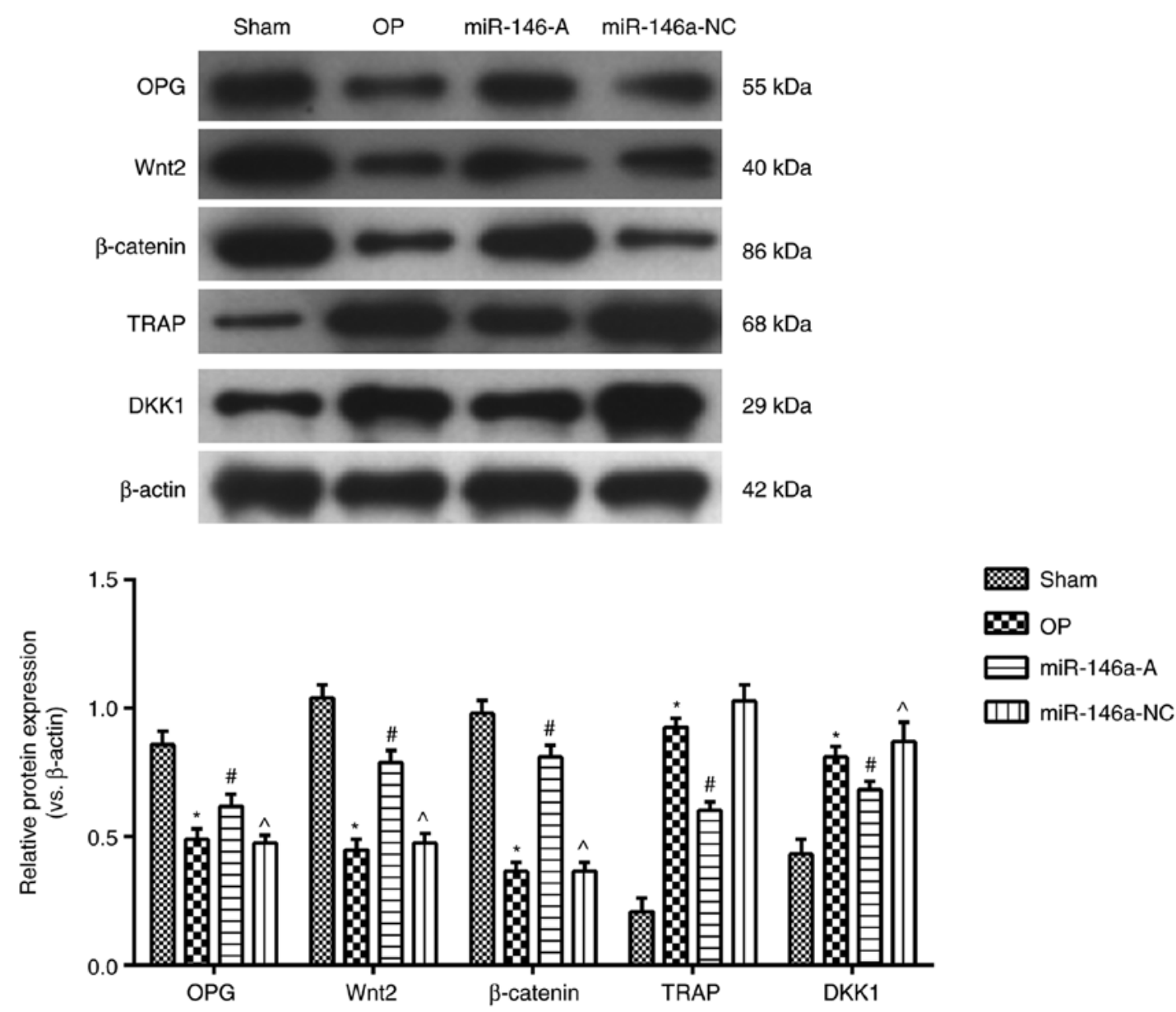

Figure 4. Effects of miR-146a on the expression of OPG, TRAP, DKK1 and Wnt2, $\beta$-catenin proteins in jawbone tissues. * $<<0.05$, compared with the sham group; ${ }^{\prime} \mathrm{P}<0.05$, compared with the OP group; ${ }^{\wedge} \mathrm{P}<0.05$, compared with the miR-146a group. miR-146a, microRNA-146a; OPG, osteoprotegerin; TRAP, tartrate-resistant acid phosphatase; DKK1, dickkopf1; sham, sham group; OP, osteoporosis group, miR-146a, miR-146a-A antagonist group; miR-146a-NC, miR-146a antagonist negative control group.

TRAP staining (Fig. 5E) indicated that the osteoclast number of the miR-146a-A and DKK2-C2 groups was significantly lower compared with that of the OP group $(\mathrm{P}<0.05)$. However, the osteoclast number in the miR + E group was significantly higher compared with that of the miR-146a-A group $(\mathrm{P}<0.05)$, whereas no significant differences were noted between the miR-146a-A and DKK2-C2 groups $(\mathrm{P}>0.05)$. These results indicated that the downregulation of miR-146a expression attenuated the pathological alterations of the jawbone via the interaction with the $\mathrm{Wnt} / \beta$-catenin signaling pathway.

miR-146a affects NFATcl, $c$-Fos and CTK protein expression by regulating the Wnt/ $\beta$-catenin signaling pathway in the jawbone. The expression levels of NFATc1, c-Fos and CTK in the jawbone were analyzed by immunohistochemistry (Fig. 6). The results indicated that the expression of NFATc1 (Fig. 6A), c-Fos (Fig. 6B) and CTK (Fig. 6C) in the other groups was significantly increased compared with that noted in the sham group $(\mathrm{P}<0.05)$. The expression levels of NFATc1, c-Fos and CTK were significantly downregulated in the miR-146a-A and DKK2-C2 groups compared with those of the OP group, $(\mathrm{P}<0.05)$. In addition, the expression levels of NFATc1, c-Fos and CTK were notably increased in the miR $+\mathrm{E}$ group compared to those in the miR-146a-A group $(\mathrm{P}<0.05)$. No significant differences were noted between the miR-146a-A and the DKK2-C2 groups with regard to the aforementioned indices $(\mathrm{P}>0.05)$. Taken together, these results indicated that the effects of miR-146-A were similar to those of DKK2-C2.

miR-146a interacts with the Wnt/ $\beta$-catenin signaling pathway in the jawbone. The expression levels of OPG, Wnt 2, $\beta$-catenin, TRAP and DKK1 in the jawbone were further analyzed in each group by western blot analysis (Fig. 7). The expression levels of OPG, Wnt 2 and $\beta$-catenin were significantly decreased $(\mathrm{P}<0.05)$, while the expression levels of TRAP and DKK1 proteins were significantly increased in the other groups compared to those of the sham group $(\mathrm{P}<0.05)$. The expression levels of OPG, Wnt2 and $\beta$-catenin proteins were notably increased, while the levels of TRAP and DKK1 proteins were significantly reduced in the miR-146a-A and DKK2-C2 groups $(\mathrm{P}<0.05)$. Furthermore, the expression levels of OPG, Wnt 2 and $\beta$-catenin proteins in the $\mathrm{miR}+\mathrm{E}$ group were significantly decreased, while those of TRAP and DKK1 proteins were markedly increased in comparison with the miR-146a-A group $(\mathrm{P}<0.05)$. The expression levels of these proteins were similar between the miR-146a-A and 

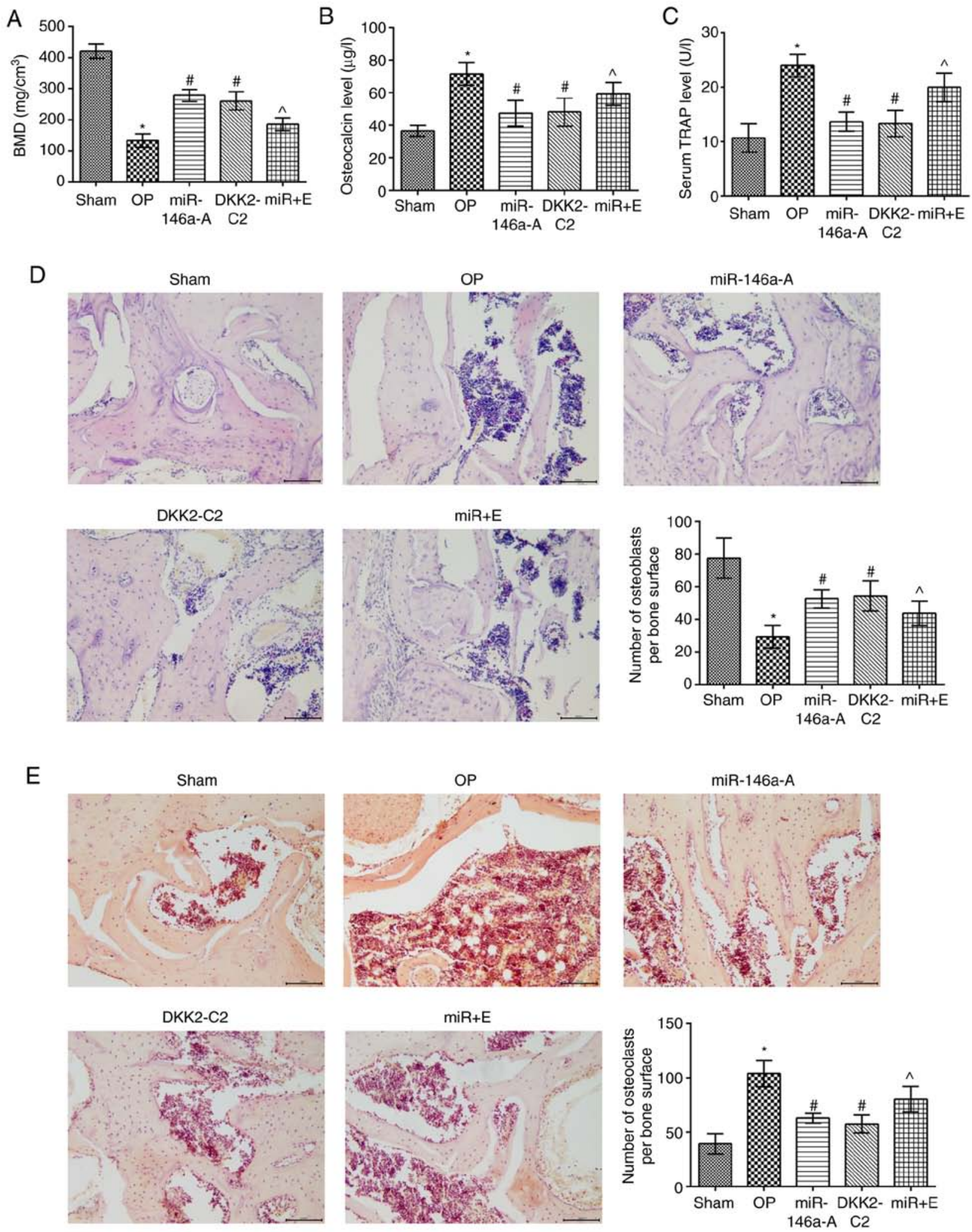

Figure 5. Activation of the Wnt/ $\beta$-catenin signaling pathway induces pathological alteration in the jaws and reduces the serum levels of osteocalcin and TRAP. (A) BMD determination; the expression levels of osteocalcin (B) and TRAP (C) were measured in the serum by ELISA. (D) The pathological alterations caused in the jaws were analyzed by H\&E staining (x200) (E). TRAP staining (x200 magnification). " $\mathrm{P}<0.05$, compared with the sham group; ${ }^{*} \mathrm{P}<0.05$, compared with the OP group; ${ }^{\wedge} \mathrm{P}<0.05$, compared with the miR-146a group. TRAP, tartrate-resistant acid phosphatase; BMD, bone mineral density; H\&E, hematoxylin-eosin; sham, sham group; OP, osteoporosis group; miR-146a-A, miR-146a antagonist group; DKK2-C2, Wnt activator group; miR + E: miR-146a antagonist + Wnt inhibitor group.

DKK2-C2 groups $(\mathrm{P}>0.05)$. Taken together, these data indicated that the downregulation of miR-146a inhibited
OP in the jaws of OVX rats by regulating the Wnt/ $\beta$-catenin signaling pathway. 
A

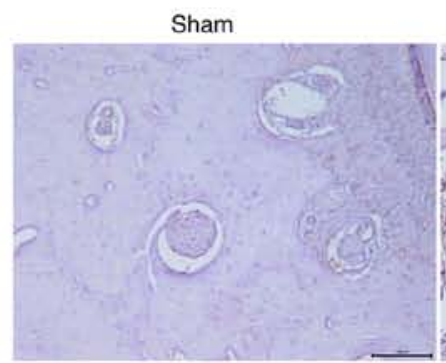

DKK2-C2

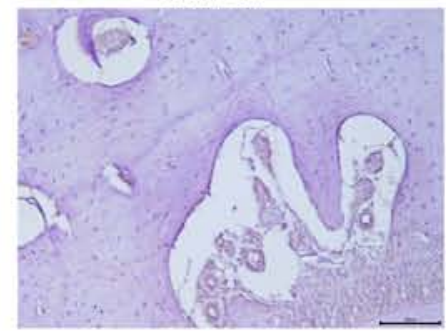

B
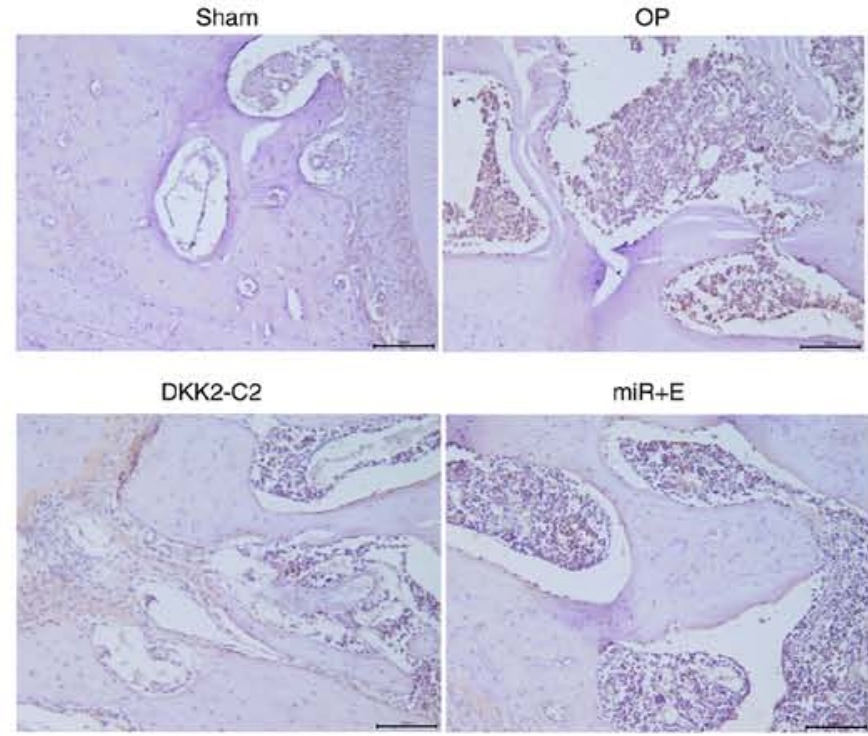

C
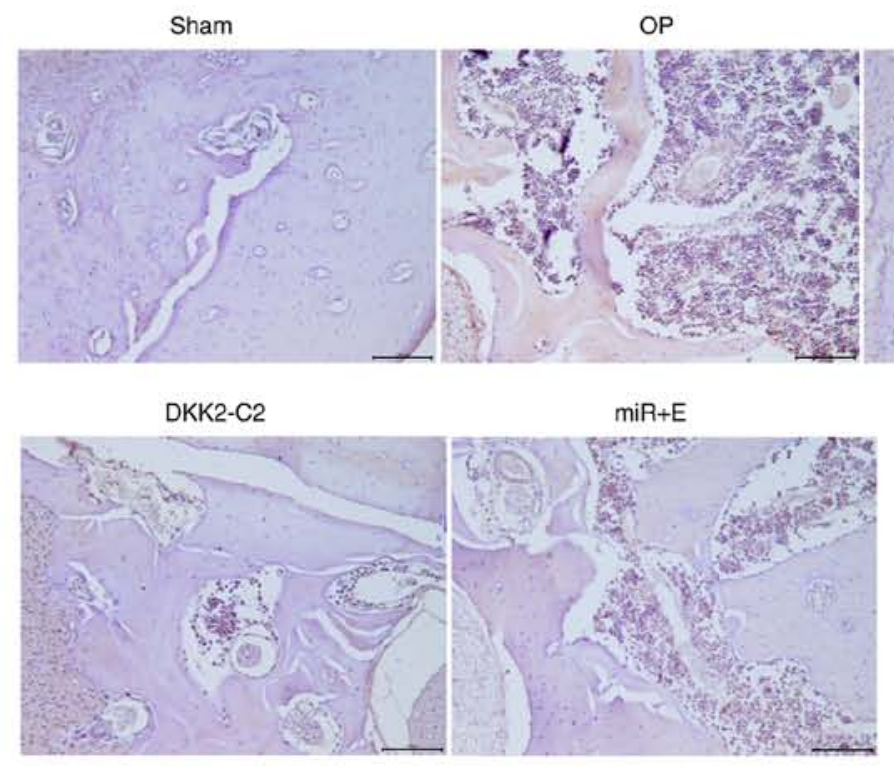

OP

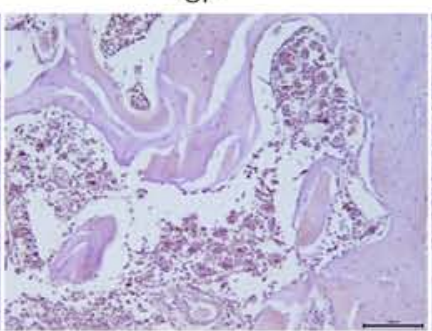

$\mathrm{miR}+\mathrm{E}$

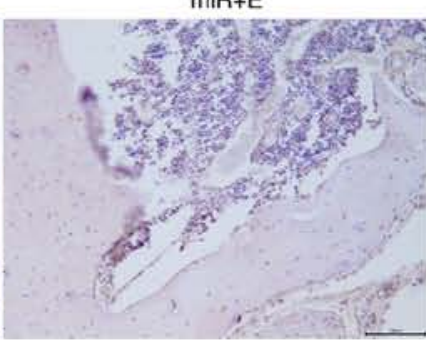

OP
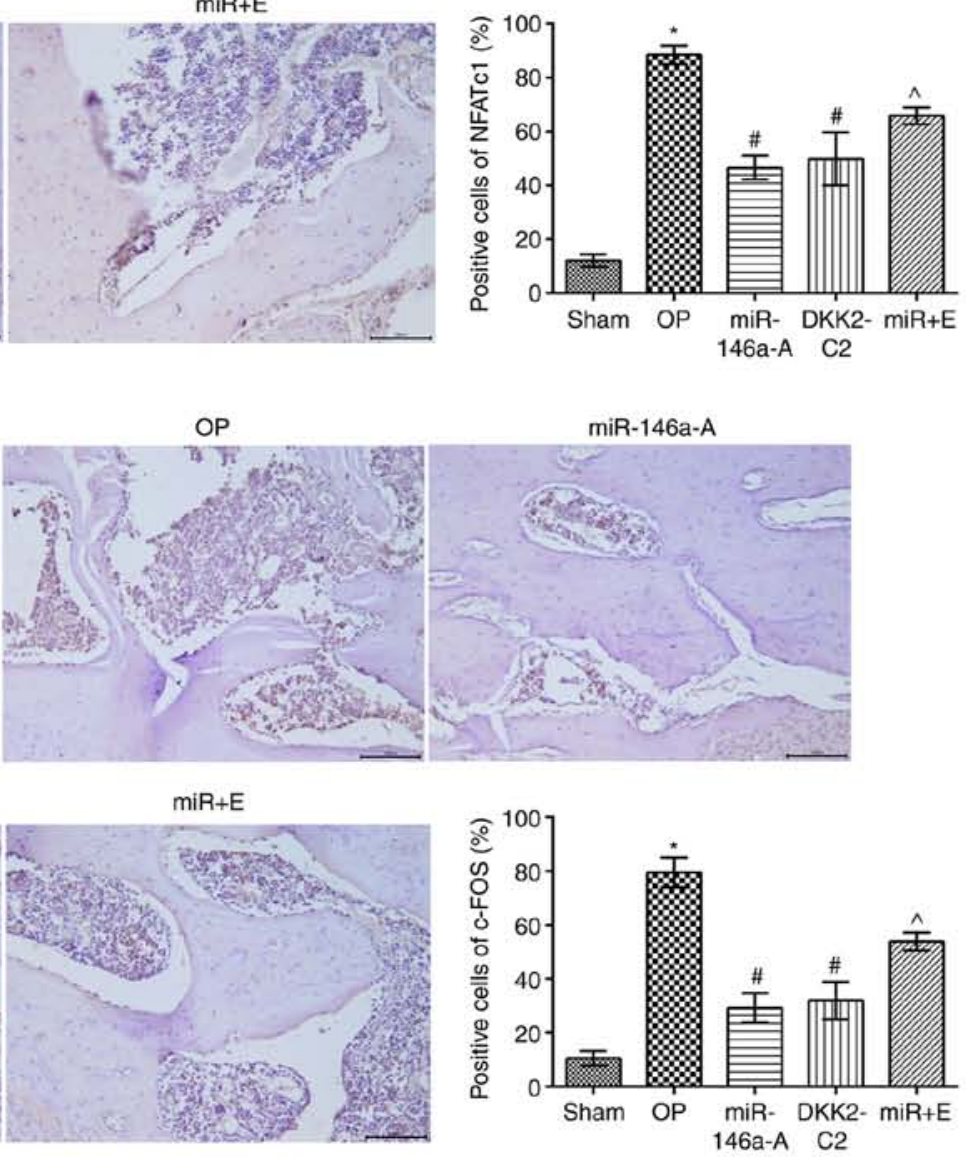

miR-146a-A

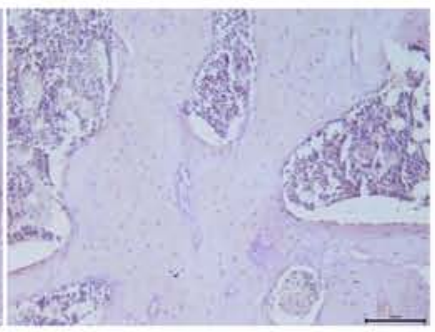

146a-A C2
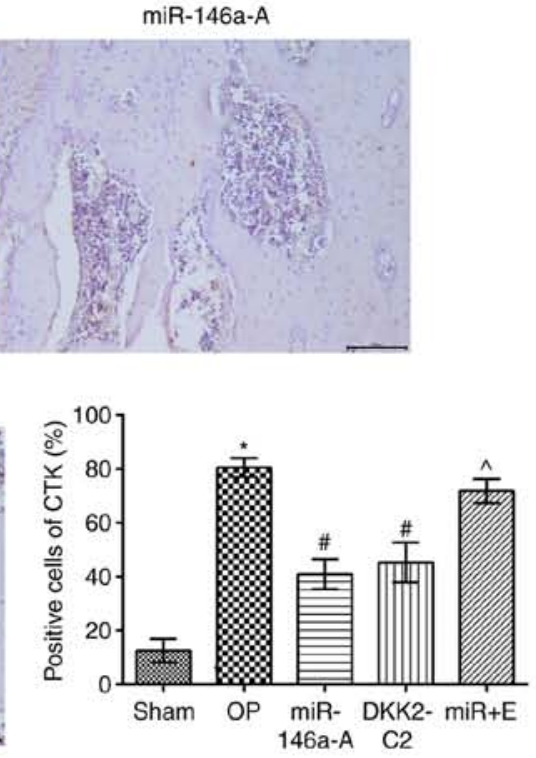

Figure 6. Activation of the Wnt/ $\beta$-catenin signaling pathway alters the expression levels of (A) NFATc1, (B) c-Fos and (C) CTK proteins in the jaws of rats. ${ }^{*} \mathrm{P}<0.05$, compared with the sham group; ${ }^{\#} \mathrm{P}<0.05$, compared with the OP group; ${ }^{\wedge} \mathrm{P}<0.05$, compared with the miR-146a group. NFATc1, nuclear factor of activated T cells c1; CTK, cathepsin K; sham, sham group; OP, osteoporosis group; miR-146a-A, miR-146a antagonist group; DKK2-C2, Wnt activator group; miR + E, miR-146a antagonist + Wnt inhibitor group. 

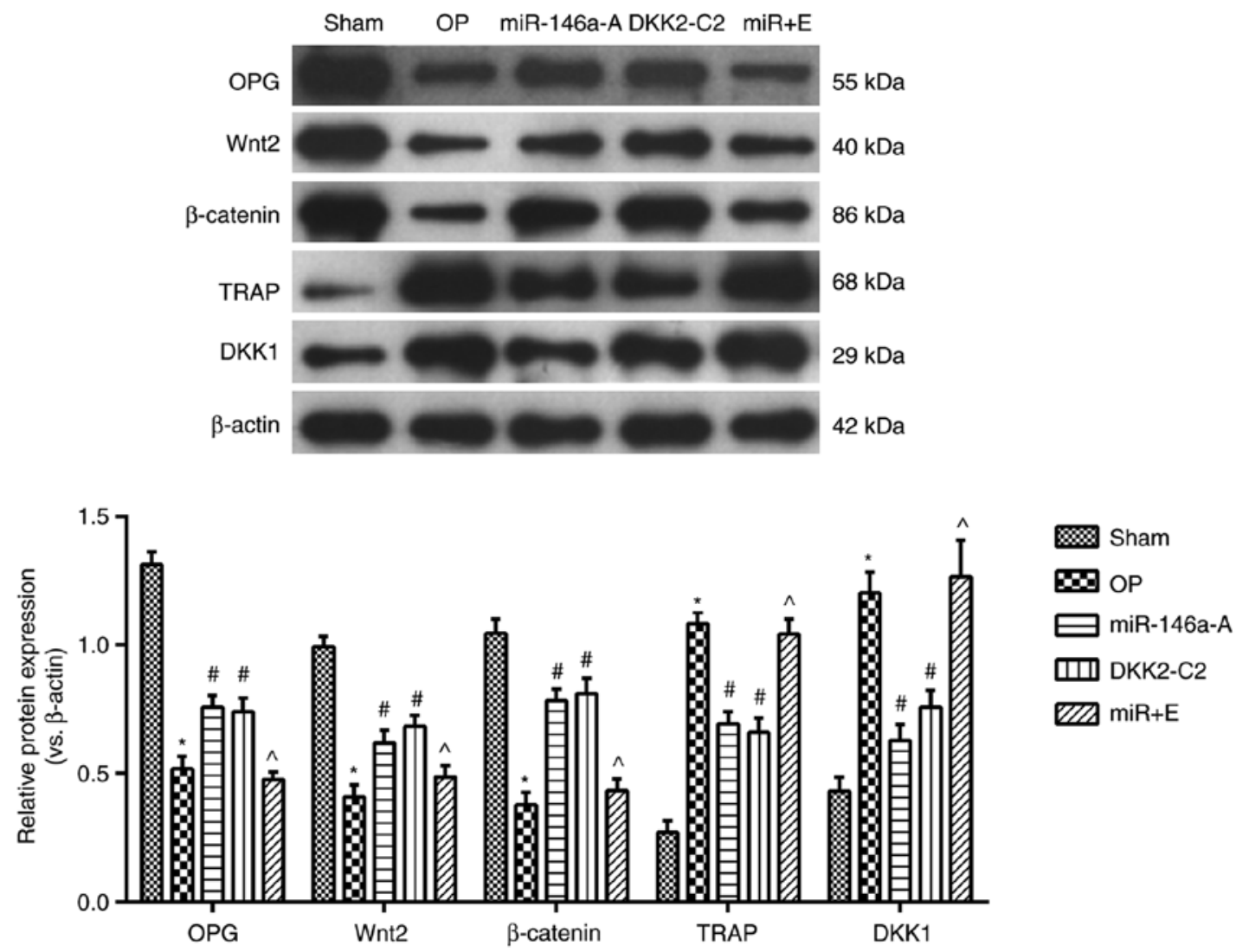

Figure 7. Activation of the Wnt/ $\beta$-catenin signaling pathway alters the expression levels of OPG, TRAP, DKK1, Wnt 2 and $\beta$-catenin proteins in the jaw. ${ }^{*} \mathrm{P}<0.05$, compared with the sham group; ${ }^{*} \mathrm{P}<0.05$, compared with the OP group; ${ }^{\wedge} \mathrm{P}<0.05$, compared with the miR-146a group. OPG, osteoprotegerin; TRAP, tartrate-resistant acid phosphatase; DKK1, dickkopf1; sham, sham group; OP, osteoporosis group; miR-146a-A, miR-146a antagonist group; DKK2-C2, Wnt activator group; miR + E, miR-146a antagonist + Wnt inhibitor group.

\section{Discussion}

OP is a bone disease caused by metabolic disturbance, which is characterized by a decrease in BMD and microstructure abnormalities of the bone tissue (20). Several studies have demonstrated that the impairment in the balance of bone marrow stem cell differentiation into osteoblasts and osteoclasts is the main cause of OP (21-23). In the present study, the results indicated that the downregulation of miR-146a improved BMD and increased bone formation markers in OVX rats, whereas the results confirmed that downregulation of miR-146a inhibited OP in OVX rats by activating the $\mathrm{Wnt} / \beta$-catenin signaling pathway.

miR-146a is associated with the development of RA by osteoclasts and synovium fibroblasts (24). Zhao et al (24) demonstrated that miR-146a played a key role in OP induced by estrogen deficiency. miR-146a knockout protected bone loss in OVX mice by increasing OPG and decreasing TRAP levels (24). In the present study, the results demonstrated that the downregulation of miR-146a increased OPG and reduced TRAP expression in OVX rats. The data further confirmed the negative role of miR-146a in OVX rats. In addition, c-Fos is indispensable for the activation of NFATc1, which translocates into the nucleus and adjusts the expression levels of genes associated with osteoclast differentiation and function, such as TRAP and CTK (25). The present study indicated that downregulation of miR-146a reduced the expression levels of NFATc1, c-Fos and CTK.

Furthermore, DKK1 can inhibit the classical Wnt signaling pathway by binding to the Wnt-receptor LRP5 (26). In the present study, the downregulation of miR-146a promoted osteogenic differentiation and inhibited DDK1 expression. The $\mathrm{Wnt} / \beta$-catenin signaling pathway plays an important role in OP $(16,18)$. $\beta$-catenin accumulates in the nucleus and binds to enhancer-binding transcription factor-1 in order to promote the expression of Runx2, which is one of the Wnt target genes and elicits a series of reactions, such as the proliferation and differentiation of osteoblasts $(27,28)$. In the present study, the Wnt2 activator and inhibitor were used to confirm that miR-146a regulates OP in OVX rats via the $W n t / \beta$-catenin pathway. The results indicated that the effects of the Wnt 2 activator were similar to those noted by the downregulation of miR-146a. This demonstrated that miR-146a inhibited OP in OVX rats by activating the $\mathrm{Wnt} / \beta$-catenin signaling pathway.

In conclusion, the present study investigated the role of miR-146a on OP in the jaws of OVX rats. The results indicated that downregulation of miR-146a could inhibit OP in castrated rats by activating the $\mathrm{Wnt} / \beta$-catenin signaling pathway. In addition, the results provided novel insight with regard to the role of miR-146a in the induction of OP in the jawbone of OVX rats and can be used further for the development of a potential therapeutic strategy for OP. 


\section{Acknowledgements}

Not applicable.

\section{Funding}

Not applicable.

\section{Availability of data and materials}

All data generated or analyzed during this study are included in this published article or are available from the corresponding author on reasonable request.

\section{Authors' contributions}

HL and XY carried out the experimental work and the data collection and interpretation, participated in the design and coordination of the experimental work, the acquisition of the data, and in the preparation of the manuscript. GZ participated in the data collection, analysis of the data. All authors read and approved the final manuscript.

\section{Ethics approval and consent to participate}

The animal experiments adhered to the NIH guidelines (NIH Pub. no. 85-23, revised 1996) and were approved by the Animal Protection and Use Committee of the 960th Hospital of the PLA Joint Logistics Support Force.

\section{Patient consent for publication}

Not applicable.

\section{Competing interests}

The authors declare that they have no competing interests.

\section{References}

1. Martel-Pelletier J, Barr AJ, Cicuttini FM, Conaghan PG, Cooper C, Goldring MB, Goldring SR, Jones G, Teichtahl AJ and Pelletier JP: Osteoarthritis. Nat Rev Dis Primers 2: 16072, 2016

2. Glyn-Jones S, Palmer AJ, Agricola R, Price AJ, Vincent TL, Weinans H and Carr AJ: Osteoarthritis. Lancet 386: 376-387, 2015.

3. Loeser RF, Goldring SR, Scanzello CR and Goldring MB: Osteoarthritis: A disease of the joint as an organ. Arthritis Rheum 64: 1697-1707, 2012.

4. Van Spil WE, Kubassova O, Boesen M, Bay-Jensen AC and Mobasheri A: Osteoarthritis phenotype novel therapeutic targets. Biochem Pharmacol 165: 41-48, 2019.

5. O'Neill TW, McCabe PS and McBeth J: Update on the epidemiology, risk factors and disease outcomes of osteoarthritis. Best Pract Res Clin Rheumatol 32: 312-326, 2018.

6. Kalaitzoglou E, Griffin TM and Humphrey MB: Innate immune responses and osteoarthritis. Curr Rheumatol Rep 19: 45, 2017.

7. Kim JR, Jong Y and Hyun K: Therapeutics in osteoarthritis based on an Understanding of its molecular pathogenesis. Int J Mol Sci 19: 674, 2018.

8. Herrero-Beaumont G, Pérez-Baos S, Sánchez-Pernaute O, Roman-Blas JA, Lamuedra A and Largo R: Targeting chronic innate inflammatory pathways, the main road to prevention of osteoarthritis progression. Biochem Pharmacol 165: 24-32, 2019.
9. Gómez R, Villalvilla A, Largo R, Gualillo $\mathrm{O}$ and Herrero-Beaumont G: TLR4 signaling in osteoarthritis-finding targets for candidate DMOADs. Nat Rev Rheumatol 11: 159-170, 2014.

10. Rachner TD, Khosla S and Hofbauer LC: Osteoporosis: Nowand the future. Lancet 377: 1276-1287, 2011.

11. Black DM and Rosen CJ: Clinical practice. Postmenopausal osteoporosis. N Engl J Med 374: 254-262, 2016.

12. Manolagas SC and Jilka RL: Bone marrow, cytokines, and bone remodeling. Emerging insights into the pathophysiology of osteoporosis. N Engl J Med 332: 305-311, 1995.

13. Sobacchi C, Schulz A, Coxon FP, Villa A and Helfrich MH: Osteopetrosis: Genetics, treatment and new insights into osteoclast function. Nat Rev Endocrinol 9: 522-536, 2013.

14. Almeida M, Laurent MR, Dubois V, Claessens F, O'Brien CA, Bouillon R, Vanderschueren D and Manolagas SC: Estrogens and androgens in skeletal physiology and pathophysiology. Physiol Rev 97: 135-187, 2017.

15. Khosla S, Oursler MJ and Monroe DG: Estrogen and the skeleton. Trends Endocrinol Metab 23: 576-581, 2012.

16. Choi HK, Kim GJ, Yoo HS, Song DH, Chung KH, Lee KJ, Koo YT and An JH: Vitamin C activates osteoblastogenesis and inhibits osteoclastogenesis via Wnt/ $\beta$-catenin/ATF4 signaling pathways. Nutrients 11: 506, 2019.

17. Lee KY, Kim JH, Kim EY, Yeom M, Jung HS and Sohn Y: Water extract of Cnidii Rhizoma suppresses RANKL-induced osteoclastogenesis in RAW 264.7 cell by inhibiting NFATc1/c-Fos signaling and prevents ovariectomized bone loss in SD-rat. BMC Complement Altern Med 19: 207, 2019.

18. Liu TJ and Guo JL: Overexpression of microRNA-141 inhibits osteoporosis in the jawbones of ovariectomized rats by regulating the Wnt/ $\beta$-catenin pathway. Arch Oral Biol 113: 104713, 2020.

19. Livak KJ and Schmittgen TD: Analysis of relative gene expression data using real-time quantitative PCR and the 2(-Delta Delta C(T)) method. Methods 25: 402-408, 2001.

20. Ji X, Chen X and Yu X: MicroRNAs in osteoclastogenesis and function: Potential therapeutic targets for osteoporosis. Int J Mol Sci 17: 349, 2016.

21. Chou CH, Shrestha S, Yang CD, Chang NW, Lin YL, Liao KW, Huang WC, Sun TH, Tu SJ, Lee WH, et al: miRTarBase update 2018: A resource for experimentally validated microRNA-target interactions. Nucleic Acids Res 46: D296-D302, 2018

22. Ashburner M, Ball CA, Blake JA, Botstein D, Butler H, Cherry JM, Davis AP, Dolinski K, Dwight SS, Eppig JT, et al: Gene ontology: Tool for the unifcation of biology. Te Gene Ontology Consortium. Nat Genet 25: 25-29, 2000.

23. David C, Mundo AF, Haw R, Milacic M, Weiser J, Wu G, Caudy M, Garapati P, Gillespie M, Kamdar MR, et al: The Reactome pathway knowledgebase. Nucleic Acids Res 42 (Database issue): D472-D477, 2014.

24. Zhao J, Huang M, Zhang X, Xu J, Hu G, Zhao X, Cui P and Zhang X: miR-146a deletion protects from bone loss in OVX mice by suppressing RANKL/OPG and M-CSF in bone microenvironment. J Bone Miner Res 34: 2149-2161, 2019.

25. Kim JH, Kim EY, Lee B, Min JH, Song DU, Lim JM, Eom JW, Yeom M, Jung HS and Sohn Y: The effects of Lycii Radicis Cortex on RANKL-induced osteoclast differentiation and activation in RAW 264.7 cells. Int J Mol Med 37: 649-658, 2016.

26. Yao GQ, Troiano N, Simpson CA and Insogna KL: Selective deletion of the soluble colony-stimulating factor 1 isoform in vivo prevents estrogen-deficiency bone loss in mice. Bone Res 5: 17022, 2017.

27. Hu L, Su P, Yin C, Zhang Y, Li R, Yan K, Chen Z, Li D, Zhang G, Wang L, et al: Microtubule actin crosslinking factor 1 promotes osteoblast differentiation by promoting $\beta$-catenin/TCF1/Runx 2 signaling axis. J Cell Physiol 233: 1574-1584, 2018.

28. Kahler RA and Westendorf JJ: Lymphoid enhancer factor-1 and beta-catenin inhibit Runx2-dependent transcriptional activation of the osteocalcin promoter. J Biol Chem 278: 11937-11944, 2003.

This work is licensed under a Creative Commons Attribution-NonCommercial-NoDerivatives 4.0 International (CC BY-NC-ND 4.0) License. 